\title{
Transcatheter transseptal mitral valve implantation with Edwards SAPIEN 3: the first experience of a single center
}

\author{
Paweł Kralisz ${ }^{1}$, Marek Frank², Bożena Sobkowicz ${ }^{3},{\text { Sławomir Dobrzycki }{ }^{1} \text {, Tomasz Hirnle }}^{2}$ \\ ${ }^{1}$ Department of Invasive Cardiology, University Hospital, Bialystok, Poland \\ ${ }^{2}$ Department of Cardiosurgery, University Hospital, Bialystok, Poland \\ ${ }^{3}$ Department of Cardiology, University Hospital, Bialystok, Poland
}

Adv Interv Cardiol 2021; 17, 1 (63): 105-108

DOI: https://doi.org/10.5114/aic.2021.104776

\section{Introduction}

In recent years, there has been a clear trend towards increased mitral bioprosthetic valve surgery in comparison to mechanical prostheses [1]. According to the ESC Guidelines mitral bioprostheses are recommended for patients > 70 years old or those with a life expectancy lower than the presumed durability of the bioprosthesis (class IIa/C). Importantly, the guidelines also recognize the prominence of quality of life after cardiosurgery and focus on patients' preferences (class Ic), especially if long-term anticoagulation is nonoptimal (class Ic) [2]. Structural deterioration of mitral bioprostheses (structural valve deterioration - SVD) is the main limitation for their use. The average lifespan of a bioprosthetic valve is estimated at 16 years and the reoperation due to SVD affects as many as $75 \%$ of patients after a 20 -year follow-up [3, 4]. Reoperation is usually associated with high risk of complications and mortality $[5,6]$.

The alternative option is transcatheter mitral valve implantation (TMVI), especially in patients with high surgical risk. TMVI is indicated in patients with a degenerated mitral bioprosthesis or mitral regurgitation after mitral annuloplasty rings. The results of TMVI have been reported in registries and single-center or case reports [7-10]. Technically, TMVI is feasible via a transapical approach or through venous access and atrial septum puncture. The transapical approach has been dominant in TMVI as it was well tried in transcatheter aortic valve implantation (TAVI) procedures. The transseptal approach is technically more demanding yet due to lesser invasiveness it may lead to superior early and late treatment effects [8]. The latest American registry STS/ACC/ TVT reports similar prevalence of TMVI procedures with both approaches [7].

\section{Material and methods}

We performed $5 \mathrm{TMVI}$ procedures in our center in January to September 2020. All patients were qualified for the procedure during Heart Team assembly. All but 1 patient were at a high cardiovascular risk. Baseline characteristics of patients are presented in Table I.

Procedures were performed in the hybrid operating room with cooperation of specialists from the Department of Cardiosurgery and Invasive Cardiology at the University Hospital in Bialystok.

All procedures were performed under general anesthesia with fluoroscopic and transesophageal echocardiographic (TEE) guidance. The right radial artery was cannulated for systemic pressure monitoring. The right jugular vein served for temporary pacemaker insertion. The first patient had additional left femoral artery cannulation with a pigtail catheter introduced to the left ventricle for mitral imaging during ventriculography and pressure gradient measurement. Due to the equivocal usefulness of such a maneuver (pressure gradient in the left ventricular outflow tract (LVOT) in TEE proved satisfactory), we dropped it in subsequent patients. The $6 \mathrm{Fr}$ sheath in the right femoral vein was exchanged for a $8.5 \mathrm{Fr}$ sheath for transseptal puncture (Swartz Braided Transseptal Guiding Introducer, Abbott). Transseptal puncture was performed with a transseptal needle (BRK-1 XS Transseptal Needle, Abbott) under TEE guidance. Half of the heparin dose was administered before the transseptal puncture; the full dose of $100 \mathrm{lU} / \mathrm{kg}$ was completed after the left atrium was reached with the target ACT 300-350 s during the procedure. The first patient had a transseptal puncture in the upper-posterior location. This location did not seem to be favorable in the next 2 patients and the postero-inferior location appeared to

Corresponding author:

Paweł Kralisz MD, PhD, Department of Invasive Cardiology, University Hospital, Bialystok, Poland, e-mail: paqral@yahoo.com

Received: 23.11.2020, accepted: 19.12.2020. 
Table I. Basic characteristics

\begin{tabular}{|c|c|c|c|c|c|c|c|c|c|}
\hline $\begin{array}{l}\text { Patient } \\
\text { (age, sex) }\end{array}$ & $\begin{array}{l}\text { Time from } \\
\text { first mitral } \\
\text { surgery } \\
\text { [years] }\end{array}$ & NYHA & $\begin{array}{c}\text { GFR } \\
<50 \mathrm{ml}\end{array}$ & CABG & $\mathrm{PCl}$ & $\begin{array}{c}\text { PSAP } \\
>50 \mathrm{~mm} \mathrm{Hg}\end{array}$ & $\mathrm{AF}$ & EF LK (\%) & $\begin{array}{c}\text { EuroSCORE } \\
\text { II }\end{array}$ \\
\hline $69 / F$ & 7 & III & $(+)$ & $(+)$ & $(-)$ & $(-)$ & $(-)$ & 60 & 8.62 \\
\hline $80 / F$ & 12 & ॥ & $(-)$ & $(+)$ & $(+)$ & $(-)$ & $(-)$ & 65 & 12.1 \\
\hline $69 / F$ & 9 & III & $(-)$ & $(-)$ & $(-)$ & $(-)$ & $(+)$ & 50 & 8.47 \\
\hline $72 / M$ & 9 & II & $(-)$ & $(+)$ & $(+)$ & $(-)$ & $(-)$ & 25 & 10.15 \\
\hline $77 / F$ & 4 & II & $(-)$ & $(-)$ & $(-)$ & $(+)$ & $(+)$ & 70 & 3.36 \\
\hline
\end{tabular}

facilitate navigation in the left atrium in the fourth and fifth patient. With detailed analysis of initial computed tomography (CT) and TEE images prior the procedure, the choice of optimal location of the puncture was much easier. Afterwards the diagnostic catheter 6 FR JR or IM was introduced via the sheath for transseptal puncture (mother and child technique), which allowed direction at the mitral valve and exchanging the standard guidewire for a stiffer one. Crossing the mitral valve was performed with a hydrophilic guidewire 0.035 " (Terumo) which was exchanged for an Amplatz Super Stiff (Boston Scientific, Marlborough, MA). The interatrial septum was dilated with a 12-18 $\mathrm{mm}$ peripheral balloon of $4 \mathrm{~cm}$ length; $14 \mathrm{~mm}$ balloon diameter seemed to be optimal. At that time, the sheath for transseptal puncture was exchanged for the sheath to advance the valve. It is worth emphasizing that the valve needed to be mounted similarly as in the transapical TAVI (reversely to transfemoral TAVI). In all cases a Sapien 3 valve was implanted. The alignment of the valve on the balloon was performed in the vein with the tip of the delivery system at the level of the right atrium. Having positioned the THV in the mitral valve area, adequate THV location was confirmed with fluoroscopic and TEE guidance. The C-arm rotation of 30 degrees (RAO) enabled long-axis mitral valve visualization. Of key importance in THV positioning was to avoid projection of the mitral bioprosthesis at the ventricular site (which could cause LVOT obstruction) and coaxial implantation. The first task was almost effortless to achieve in fluoroscopy with a stented bioprosthesis. It was far more complicated with a prosthesis which could not be visualized in X-ray ( 3 patients). In those cases the absence of any angiographic markers was substituted with TEE guidance to achieve appropriate THV positioning. TEE was performed with the Philips Epiq $7 \mathrm{CVx} 3 \mathrm{D}$ with TEE probe X8-2t with 3D. XPlane Live mode was used for both transseptal puncture and valve positioning (real-time imaging of two perpendicular 2D images). The THV was deployed with one prolonged balloon inflation under ventricular pacing at 140-160 bpm. No additional balloon inflation was needed in any patient. Anchoring, expansion and round diameter of the THV were evaluat- ed in TEE, with the focus on the LVOT gradient, paravalvular leak and mitral regurgitation. In 1 case, the LVOT gradient was $23 \mathrm{~mm} \mathrm{Hg}$, yet no leakage or mitral valve gradient was found. The temporary endocavitary pacemaker was removed after the procedure. Venous hemostasis was achieved with the "figure-of-eight suture".

\section{Results}

Post-procedural heparin infusion was administered in all patients until therapeutic INR was achieved. Patients were extubated immediately after regaining consciousness, usually after $6 \mathrm{~h}$. No complications were observed during hospitalization. Patients were discharged home 5 days after the procedure with a scheduled follow-up visit after 1 month, 3 months and 1 year. Due to epidemiological circumstances complete 1- and 3-month follow-up was impossible (echocardiographic assessment), yet the clinical condition was established via teleconsultation.

Data concerning the procedure, hospitalization, and initial 30-day follow-up are presented in Table II.

\section{Discussion}

Efficacious anchoring of the valve in the mitral annulus is of key importance during the TMVI procedure. Fundamental difficulty stems from the high pressure gradient between the left ventricle and left atrium. An indispensable condition is the absence of perivalvular leak of the mitral bioprosthesis or tearing of the mitral annulus after annuloplasty. Properly sutured surgical prostheses and mitral annuli usually constitute sufficient support for THV implantation.

A degenerated mitral bioprosthesis (valve-in-valve $\mathrm{ViV}$ ) is the optimal target for the transcatheter procedure. The dimensions of the bioprosthesis are known in advance from the producer's information, most of them are clearly visible in X-ray imaging or echo, and the stiff structure allows safe and solid deployment. Moreover, the bioprosthesis contributes to coaxial positioning of the valve. ViV procedures are generally successful and are characterized by favorable early and long-term outcome [11-13].

The alternative, less comfortable indication is valvein-ring implantation (ViR). Periprocedural complications 
include difficult sizing, positioning or deployment, which are more often connected with paravalvular leak, inappropriate sealing, the necessity of second valve implantation or obstruction of the LVOT. The contact area between the deployed THV and the annulus is smaller than in the degenerated prosthesis, which influences the stability of the device. The procedure has a two times greater early and 1-year mortality than VIV [9].

The transapical approach is convenient for several types of valves (Sapien, Lotus, Direct flow, Melody) [9]. This approach favors stable procedure controlling and precise, coaxial orientation and, if needed, repositioning of the device. By contrast, the transseptal procedure can be done only with one valve type from the Edwards SAPIEN family. This comes from the unique structure of the prosthesis and primarily the guiding system, which allows maneuvering in the left atrium.

The choice of appropriate size of the THV is primarily based on the nominal THV values provided by the manufacturer of the mitral bioprosthesis. Available apps that provide complete data of the structure and sizing of bioprostheses and mitral rings are also very helpful [14].

Procedural success applies to almost $90 \%$ of cases, and is lower in ViR. Complications are typical for transcatheter procedures, including most often vascular complications, followed by atrial or ventricular perforation and cardiac tamponade, acute kidney injury, the necessity of conversion to open heart surgery, embolization or implantation of a second THV. In $80 \%$ of patients, mitral insufficiency is absent or mild. A unique complication is LVOT obstruction. It affects $2-13 \%$ of cases, and prevails in $\operatorname{ViR}[7,9,11]$. It is defined as the increase of LVOT gradient $>30 \mathrm{~mm} \mathrm{Hg}$ at rest in echocardiographic imaging. A significant gradient is generally considered to be an LVOT gradient $>50 \mathrm{~mm} \mathrm{Hg}$. Small size of the left ventricle, small LVOT diameter, thick intraventricular septum, acute aortomitral angle, and the size and manner of THV implantation are predisposing factors. The risk of thrombosis in the THV remains unclear. Hypothetically, enlarged left atrium, atrial fibrillation, and the combination of a surgical device of foreign origin and the THV may predispose to thrombosis, yet anticoagulation is routinely indicated in such cases.

\section{Conflict of interest}

The authors declare no conflict of interest.

\section{References}

1. Gammie JS, Sheng S, Griffith BP, et al. Trends in mitral valve surgeryin the United States: results from the Society of Thoracic SurgeonsAdult Cardiac Database. Ann Thorac Surg. 2009; 87: 1431-9.

2. Baumgartner H, Falk V, Bax JJ, et al.; ESC Scientific Document Group. 2017 ESC/EACTS Guidelines for the management of valvular heart disease. Eur Heart J 2017; 38: 2739-91.
Table II. Procedural details and early results

\begin{tabular}{|c|c|}
\hline Parameter & Result \\
\hline $\begin{array}{l}\text { Complete technical procedural success (ac- } \\
\text { cording to MVARC) }\end{array}$ & $5(100 \%)$ \\
\hline Periprocedural death (within 24 h) & 0 \\
\hline Procedural duration (skin to skin) [min] & $246(200-300)$ \\
\hline Fluoroscopy time [min] & $89.9(56.4-134.8)$ \\
\hline Contrast vol. [ml] & $70(0-134)$ \\
\hline \multicolumn{2}{|l|}{ Valve size: } \\
\hline $26 \mathrm{~mm}$ & 3 \\
\hline $29 \mathrm{~mm}$ & 2 \\
\hline \multicolumn{2}{|l|}{ Complications: } \\
\hline Vascular access site bleeding complication & 0 \\
\hline Device embolization & 0 \\
\hline Need for second valve implantation & 0 \\
\hline Cardiac perforation/cardiac tamponade & 0 \\
\hline Major stroke & 0 \\
\hline New arrhythmia & 0 \\
\hline Conversion to open heart surgery & 0 \\
\hline Acute kidney injury & 0 \\
\hline Significant LVOT obstruction & 0 \\
\hline \multicolumn{2}{|l|}{$\begin{array}{l}\text { Residual mitral regurgitation after TMVI at } \\
\text { hospital discharge: }\end{array}$} \\
\hline None & 3 \\
\hline Trace or mild (MR grade I) & 2 \\
\hline Moderate or severe (MR grade $>2$ ) & 0 \\
\hline In-hospital mortality rate & 0 \\
\hline $\begin{array}{l}\text { In-hospital stay from TMVI to hospital } \\
\text { discharge (mean } \pm \text { SD) [days] }\end{array}$ & $5(5-7)$ \\
\hline \multicolumn{2}{|l|}{$\begin{array}{l}\text { Clinical follow-up after hospital discharge } \\
\text { 30-day: }\end{array}$} \\
\hline Mortality rate (all-cause) & 0 \\
\hline \multicolumn{2}{|l|}{ NYHA functional class at 30 days: } \\
\hline I & 3 \\
\hline$\|$ & 2 \\
\hline III/IV & 0 \\
\hline
\end{tabular}

3. Bourguignon T, Espitalier F, Pantaleon C, et al. Bioprosthetic mitral valve replacement in patients aged 65 years or younger: long-term outcomes with the Carpentier-Edwards PERIMOUNT pericardial valve. Eur J Cardiothorac Surg 2018; 54: 302-9.

4. Bourguignon T, Bouquiaux-Stablo AL, Loardi C, et al. Very late outcomes for mitral valve replacement with the Carpentier-Ed- 
wards pericardial bioprosthesis: 25-year follow-up of $450 \mathrm{im}$ plantations. J Thorac Cardiovasc Surg 2014; 148: 2004-11.

5. Jamieson WRE, Burr LH, Miyagishima RT, et al. Reoperation for bioprosthetic mitral structural failure: risk assessment. Circulation 2003; 108: II-98-102.

6. Mehaffey HJ, Hawkins RB, Schubert S, et al. Contemporary outcomes in reoperative mitral valve surgery. Heart 2018; 104: 652-6.

7. Guerrero M, Vemulapalli S, Xiang Q, et al. Thirty-day outcomes of transcatheter mitral valve replacement for degenerated mitral bioprostheses (valve-in-valve), failed surgical rings (valve-inring), and native valve with severe mitral annular calcification (valve-in-mitral annular calcification) in the United States: data from the Society of Thoracic Surgeons/American College of Cardiology/Transcatheter Valve Therapy Registry. Circ Cardiovasc Interv 2020; 13: e008425.

8. Eleid MF, Whisenant BK, Cabalka AK, et al. Early outcomes of percutaneous transvenous transseptal transcatheter valve implantation in failed bioprosthetic mitral valves, ring annuloplasty, and severe mitral annular calcification. JACC Cardiovasc Interv 2017; 10: 1932-42.

9. Yoon SH, Whisenant BK, Bleiziffer S, et al. Outcomes of transcatheter mitral valve replacement for degenerated bioprostheses, failed annuloplasty rings, and mitral annular calcification. Eur Heart J 2019; 40: 441-51.

10. Guerrero M, Dvir D, Himbert D, et al. Transcatheter mitral valve replacement in native mitral valve disease with severe mitral annular calcification: results from the first multicenter global registry. JACC Cardiovasc Interv 2016; 9: 1361-71.

11. Urena $M$, Brochet E, Lecomte $M$, et al. Clinical and haemodynamic outcomes of balloon-expandable transcatheter mitral valve implantation: a 7-year experience. Eur Heart J 2018; 39: 2679-89.

12. Cheung A, Webb JG, Barbanti M, et al. 5-year experience with transcatheter transapical mitral valve-in-valve implantation for bioprosthetic valve dysfunction. J Am Coll Cardiol 2013; 61: 1759-66.

13. Ye J, Cheung A, Yamashita M, et al. Transcatheter aortic and mitral valve-in-valve implantation for failed surgical bioprosthetic valves: an 8-year single centre experience. J Am Coll Cardiol Intv 2015; 8: 1735-44.

14. Bapat V. An instant guide to Valve in Valve procedures for clinicians. UBQO Ltd. http://www.ubqo.com/vivmitral. 